\title{
Ação da melatonina sobre a dinâmica sanguínea de ratas prenhes e sobre a histogênese do baço e do timo da prole
}

\author{
[The action of melatonin on the blood dynamics of pregnant rats and on the histogenesis of the \\ spleen and thymus of offspring]
}

\author{
A.C.C. Araújo, V. Wanderley-Teixeira, P.E.A. Vilaça-Junior, A.F. Soares, \\ A.J.J.M. Lemos, F.C.A. Silva, A.A.C. Teixeira*
}

Universidade Federal Rural de Pernambuco - UFRPE - Recife, PE

\begin{abstract}
RESUMO
Investigou-se a influência da melatonina sobre o hemograma de ratas prenhes e dos filhotes e sobre a histogênese e morfometria do baço e do timo dos filhotes. A melatonina foi administrada na dose $0,5 \mathrm{mg} / \mathrm{kg}$ de peso corporal, dissolvida em $0,1 \mathrm{~mL}$ de etanol e diluída em $0,3 \mathrm{~mL}$ de solução salina. Para análise do hematócrito, contagem total de hemácias e contagem total e diferencial dos leucócitos, amostras de sangue foram coletadas no sétimo, $14^{\mathrm{o}}$ e $21^{\mathrm{o}}$ dias de prenhez e aos 10 dias de nascimento dos filhotes. Cortes histológicos do baço e do timo da prole foram utilizados para histoquímica e morfometria. A ausência da melatonina promoveu alterações no hemograma apenas no terço final da gestação, sem interferir no hemograma dos filhotes, e induziu modificações morfológicas e morfométricas no timo e no baço nos primeiros dias de vida dos filhotes. Concluiu-se que a melatonina materna é importante para a modulação do hemograma em ratas prenhes e para o desenvolvimento normal do baço e do timo dos filhotes.
\end{abstract}

Palavras-chave: rato, melatonina, hemograma, prenhez, órgãos de defesa, imunidade

\begin{abstract}
We investigated the influence of melatonin on the hemogram of pregnant rats and puppies, and on the histogenesis and morphology of the spleen and thymus of puppies. Melatonin was administered at a dose $0.5 \mathrm{mg} / \mathrm{kg}$ body weight, dissolved in $0.1 \mathrm{~mL}$ of ethanol and diluted in $0.3 \mathrm{~mL}$ of saline. For hematocrit analysis, total erythrocyte count and total and differential leukocyte count, blood samples were collected on the 7th, 14th and 21st days of pregnancy and the offspring at 10 days of birth. Histological sections of spleen and thymus of the offspring were used for histochemistry and morphometry. The absence of melatonin promoted changes in blood count only in the final third of gestation, without interfering with the hemogram of the puppies, and induced morphological and morphometric changes in the thymus and spleen in the first days of life of the puppies. It was concluded that maternal melatonin is important for the modulation of the blood count in pregnant rats and the normal development of the spleen and thymus of the offspring.
\end{abstract}

Keywords: rat, melatonin, hemogram, pregnancy, defense organs, immunity

\section{INTRODUÇÃO}

Durante a gestação em mamíferos, ocorre uma ampla modulação da função tanto do sistema imune inato quanto do humoral (Markert et al., 2005; Sargent et al., 2006). Essa imunomodulação sistêmica promove

Recebido em 26 de abril de 2011

Aceito em 5 de novembro de 2012

* Autor para correspondência (corresponding author)

E-mail: alvaro@dmfa.ufrpe.br simultaneamente uma redução da atividade imunológica celular, da tolerância a diversos antígenos, porém aumenta a resposta inflamatória (Shakhar et al., 2007). Em humanos, a imunomodulação caracteriza-se por aumento do número de leucócitos (Veenstra Van Nieuwenhoven et al., 2003), supressão da citotoxicidade das células natural killer (Baley e 
Schacter, 1985; Gabrilovac et al., 1988) e aumento do número de células $\mathrm{T}$ regulatórias (Somerset et al., 2004).

A modulação do sistema imune pode ser afetada por diversos fatores, inclusive pela presença de hormônios, como é o caso da melatonina, hormônio produzido pela pineal durante o período noturno, o qual parece estimular o sistema imunológico, aumentando a mobilidade e a atividade das células de defesa, promovendo a formação dos anticorpos e, consequentemente, aumentando as defesas contra microrganismos (Claustrat et al., 2005).

Na gestação, a produção e a concentração sérica de melatonina mostram mudança gradual e atingem valores elevados no final desse período, sugerindo que esse hormônio desempenhe importante papel na manutenção da gestação (Bishnupuri e Haldar, 2001; Nakamura et al., 2001). Essa indoleamina lipofílica atravessa a placenta livremente sem ser alterada, atinge a circulação fetal com facilidade e fornece informações fotoperiódicas para o feto. Assim, influencia o fotoperíodo pré-natal por meio dos receptores desse hormônio em diversos tecidos, mediando as interações fisiológicas fetais, entre elas as funções imunológicas (Goldman, 2003).

Estudos mostram que ratos submetidos à administração de melatonina pela via oral apresentam aumento na atividade proliferativa de linfócitos, na síntese de DNA em linfócitos tímicos e esplênicos e na produção de citocinas por macrófagos em ratos portadores de tumor (El-Sokkary et al., 2003). Além disso, o fotoperíodo, a pinealectomia e a melatonina exógena podem afetar o peso e as funções do timo e do baço em mamíferos e aves (Pertsov, 2006; Wronka et al., 2008).

As interações entre a melatonina e o sistema imunológico têm sido bastante estudadas, e em praticamente todos os casos, a melatonina mostrou ter efeitos imunomoduladores da resposta imune (Esquifino et al., 2004; Faas et al., 2005). Entretanto, não é conhecido o papel desempenhando por esse hormônio durante a gestação, sobre a imunidade celular. Assim, a pesquisa investigou a influência da melatonina sobre o hemograma de ratas prenhes e dos filhotes, além de analisar a histogênese e a morfometria do baço e do timo dos filhotes.

\section{MATERIAL E MÉTODOS}

Foram utilizadas 40 ratas albinas (Rattus norvegicus albinus) com 90 dias de idade. Todo protocolo experimental foi aprovado pela Comissão de Ética institucional $\left(\mathrm{n}^{\circ}\right.$. 23082.010380/2010). As fêmeas foram distribuídas, ao acaso, em quatro grupos: o grupo I foi composto por ratas acasaladas e mantidas em ciclo claro:escuro de 12:12h (controle); o grupo II, por ratas sham-pinealectomizadas, acasaladas e mantidas em ciclo claro:escuro de 12:12h; o grupo III, por ratas pinealectomizadas, acasaladas, mantidas em ciclo claro:escuro de $12: 12 \mathrm{~h}$ e tratadas com placebo; e o grupo IV, por ratas pinealectomizadas, acasaladas, mantidas em ciclo claro:escuro de $12: 12 \mathrm{~h}$ e tratadas com melatonina.

A pinealectomia foi realizada seguindo a metodologia de Kuszak e Rodin (1977). Para isso, as fêmeas foram previamente anestesiadas com hidrocloridrato de cetamina $(80 \mathrm{mg} / \mathrm{kg})$ em associação com xilazina $(20 \mathrm{mg} / \mathrm{kg})$, por via intramuscular. $\mathrm{O}$ mesmo procedimento foi realizado com as fêmeas do grupo II (shampinealectomizadas), porém sem a retirada da glândula pineal.

A melatonina (Sigma Chemical Co., St. Louis, Mo., USA) foi administrada na dosagem de $0,5 \mathrm{mg} / \mathrm{kg}$, dissolvida em $0,1 \mathrm{~mL}$ de etanol, diluída em $0,3 \mathrm{~mL}$ de solução salina (Subramanian et al., 2007). Como placebo, foi administrado o mesmo volume de etanol e salina.

O sangue, coletado no sétimo, $14^{\circ}$ e $21^{\circ}$ dias por meio da punção da veia lateral caudal das ratas, com retirada de $500 \mu \mathrm{L}$, foi acondicionado em tubo com anticoagulante (EDTA dipotássico). Para coleta de sangue dos filhotes, anestesiados com pentobarbital $40 \mathrm{mg} / \mathrm{kg}$, por via intraperitoneal, colheu-se o mesmo volume de sangue por punção cardíaca aos 10 dias de idade. $O$ hematócrito foi determinado pelo método do micro-hematócrito, enquanto a contagem de hemácias e leucócitos foi realizada em câmara de Neubauer (Lorenzi, 2006). Os resultados foram submetidos à fórmula: $\mathrm{Hm} / \mu \mathrm{L}=\mathrm{Hm} \times 10$ × 400 × 5, em que Hm é o número total de hemácias contadas, 10 é o fator de conservação para o volume de $1 \mathrm{~mm}^{3}$ (profundidade da câmara de Neubauer), 400 é o fator da conservação da diluição utilizada, e 5 é o 
fator de conservação para área de $1 \mathrm{~mm}^{2}$ (5 de 25 quadrados médios). Para a contagem total de leucócitos, os resultados foram submetidos à fórmula: $\mathrm{Lc} / \mu \mathrm{L}=\mathrm{Lc} \times 20 \times 10 / 4$, em que Lc é o número total de leucócitos contados, 10 é o fator de conservação para o volume de $1 \mathrm{~mm}^{3}$ (profundidade da lâmina da câmara de Neubauer), 20 é o fator de conservação da diluição utilizada, e 4 é o fator de conservação para a área de $1 \mathrm{~mm}^{2}$ (número de quadrados). Para contagem diferencial de leucócitos, usou-se esfregaço sanguíneo submetido ao método de coloração pelo kit panótico e pela técnica de sudan black 1,4\%, coloração bastante útil na diferenciação de leucemias agudas.

Para coleta do baço e do timo, três filhotes de cada fêmea dos grupos experimentais foram sacrificados, com hidrocloridrato de cetamina $(80 \mathrm{mg} / \mathrm{kg})$ e xilazina $(6 \mathrm{mg} / \mathrm{kg})$, por via intramuscular, no primeiro, quinto e $10^{\mathrm{o}}$ dias de nascidos. Os órgãos foram fixados em líquido de Boüin, processados para inclusão em parafina, e os cortes submetidos à técnica de coloração de rotina pela hematoxilina-eosina e histoquímica pelo tricrômico de Mallory, orceína e impregnação argêntica. As reações de coloração histoquímica foram classificadas como intensa $(++)$, moderada $( \pm)$ e fraca $(+)$.

Para a morfometria do baço e do timo dos filhotes, realizou-se a captura de imagens por meio de câmera de Vídeo Sony®, acoplada ao microscópio Olympus ${ }^{\circledR}$ Bx50, as quais foram submetidas ao aplicativo morfometria de linhas, calibrado em micrômetros, associado ao programa ImagemLab 2000 para Windows. No baço, foram medidos 10 corpúsculos esplênicos dos filhotes/dia de nascido, de cada grupo experimental, para determinação em micrômetros das seguintes características: área, diâmetro máximo e diâmetro mínimo (Golalipour et al., 2008). A contagem das células hematopoiéticas foi realizada utilizando-se um retículo contendo 100 pontos, acoplado a uma ocular de 10x e analisado com objetiva de 40x (Weibel et al., 1966). Foram contados os pontos que incidiram sobre as células hematopoiéticas. No timo, foram utilizadas cinco lâminas/grupo/dia de nascido e mensurados 10 lóbulos tímicos para determinação da área tímica, área cortical e área medular (Alves et al., 2006). Todas as medidas foram efetuadas mediante o sistema analisador de imagens, Image Pro-plus, acoplado a um microscópio binocular da Olympus. Os dados obtidos foram submetidos ao teste não paramétrico de Kruskal-Wallis, em que as médias foram comparadas pelo teste de Wilcoxon-Mann-Whitney $(\mathrm{P}<0,05)$.

\section{RESULTADOS}

Nas Tab. 1, 2 e 3, estão as médias e os desviospadrão do número total de hemácias, do número total de leucócitos, do percentual do hematócrito e da contagem diferencial dos leucócitos ao sétimo, $14^{\underline{0}}$ e $21^{\underline{0}}$ dias de gestação, respectivamente. Só foram verificadas diferenças significativas aos 21 dias de gestação, representadas pela redução do número total de leucócitos, do percentual do hematócrito, da porcentagem de linfócitos, e pelo aumento do percentual de neutrófilos e eosinófilos nas ratas submetidas à pinealectomia e tratadas com placebo. Na Tab. 4, estão essas mesmas características para os filhotes com 10 dias após nascimento. Não foram verificadas diferenças significativas paras os parâmetros analisados.

Tabela 1. Médias do hematócrito e contagem total e diferencial das células sanguíneas de ratas com sete dias de gestação

\begin{tabular}{cccccc}
\hline Grupos & I & II & III & IV & P \\
\hline CTH $\left(10^{6} / \mu \mathrm{L}\right)$ & $462,66 \pm 34,16$ & $464,08 \pm 59,91$ & $444,99 \pm 41,22$ & $499,16 \pm 33,38$ & 0,2046 \\
CTL $\left(10^{3} / \mu \mathrm{L}\right)$ & $199,91 \pm 48,00$ & $183,83 \pm 48,56$ & $180,58 \pm 32,97$ & $199,41 \pm 24,04$ & 0,8137 \\
Hematócrito (\%) & $46,25 \pm 3,94$ & $49,41 \pm 5,74$ & $48,83 \pm 1,57$ & $51,16 \pm 1,77$ & 0,3224 \\
Linfócitos (\%) & $67,91 \pm 2,12$ & $66,00 \pm 1,14$ & $66,50 \pm 3,98$ & $66,91 \pm 3,50$ & 0,7885 \\
Neutrófilos (\%) & $23,50 \pm 1,77$ & $22,41 \pm 1,02$ & $20,33 \pm 1,62$ & $21,58 \pm 1,66$ & 0,3688 \\
Eosinófilos (\%) & $6,00 \pm 0,43$ & $5,96 \pm 0,30$ & $7,41 \pm 1,04$ & $6,16 \pm 0,38$ & 0,2272 \\
Monócitos (\%) & $1,66 \pm 0,28$ & $1,91 \pm 0,58$ & $0,83 \pm 0,52$ & $1,33 \pm 0,38$ & 0,2163 \\
Basófilos $(\%)$ & $0,66 \pm 0,28$ & $0,41 \pm 0,38$ & $0,16 \pm 0,14$ & $0,25 \pm 0,16$ & 0,2074 \\
\hline
\end{tabular}

Não houve diferença entre os grupos $(\mathrm{P}>0,05)$ pelo teste de Wilcoxon-Mann-Whitney. $\mathrm{CTH}=$ contagem total de hemácias - CTL = contagem total de leucócitos. 
Tabela 2. Médias do hematócrito e contagem total e diferencial das células sanguíneas de ratas com 14 dias de gestação

\begin{tabular}{cccccc}
\hline Grupos & I & II & III & IV & P \\
\hline CTH $\left(10^{6} / \mu \mathrm{L}\right)$ & $476,74 \pm 28,70$ & $470,33 \pm 38,61$ & $521,99 \pm 40,00$ & $498,49 \pm 39,61$ & 0,3653 \\
CTL $\left(10^{3} / \mu \mathrm{L}\right)$ & $177,89 \pm 30,78$ & $176,82 \pm 43,40$ & $166,66 \pm 45,88$ & $185,32 \pm 30,80$ & 0,9074 \\
Hematócrito $(\%)$ & $48,08 \pm 4,16$ & $44,98 \pm 2,32$ & $43,49 \pm 2,57$ & $44,24 \pm 3,21$ & 0,2616 \\
Linfócitos $(\%)$ & $69,58 \pm 2,44$ & $65,41 \pm 1,99$ & $67,91 \pm 2,06$ & $66,16 \pm 2,32$ & 0,5683 \\
Neutrófilos $(\%)$ & $20,83 \pm 2,12$ & $24,41 \pm 2,45$ & $23,50 \pm 2,41$ & $23,08 \pm 1,81$ & 0,5953 \\
Eosinófilos (\%) & $4,75 \pm 0,90$ & $5,00 \pm 0,25$ & $6,08 \pm 0,88$ & $4,91 \pm 0,87$ & 0,1372 \\
Monócitos $(\%)$ & $1,91 \pm 0,14$ & $2,16 \pm 0,28$ & $1,33 \pm 0,52$ & $2,33 \pm 0,62$ & 0,0681 \\
Basófilos $(\%)$ & $0,50 \pm 0,25$ & $0,43 \pm 0,32$ & $0,26 \pm 0,14$ & $0,75 \pm 0,56$ & 0,4582 \\
\hline
\end{tabular}

Não houve diferença entre os grupos $(\mathrm{P}>0,05)$ pelo teste de Wilcoxon-Mann-Whitney. $\mathrm{CTH}=$ contagem total de hemácias - CTL $=$ contagem total de leucócitos.

Tabela 3. Médias do hematócrito e contagem total e diferencial das células sanguíneas de ratas com 21 dias de gestação

\begin{tabular}{cccccc}
\hline Grupos & I & II & III & IV & P \\
\hline CTH $\left(10^{6} / \mu \mathrm{L}\right)$ & $426,91 \pm 26,08$ & $424,24 \pm 35,30$ & $446,41 \pm 47,53$ & $425,66 \pm 43,15$ & 0,6082 \\
CTL $\left(10^{3} / \mu \mathrm{L}\right)$ & $323,08 \pm 19,45 \mathrm{a}$ & $334,99 \pm 14,11 \mathrm{a}$ & $147,91 \pm 15,84 \mathrm{~b}$ & $358,16 \pm 13,22 \mathrm{a}$ & 0,0160 \\
Hematócrito $(\%)$ & $44,24 \pm 4,78 \mathrm{a}$ & $43,66 \pm 4,06 \mathrm{a}$ & $34,58 \pm 2,11 \mathrm{~b}$ & $45,33 \pm 3,81 \mathrm{a}$ & 0,0364 \\
Linfócitos $(\%)$ & $69,25 \pm 0,43 \mathrm{a}$ & $67,33 \pm 1,37 \mathrm{a}$ & $37,16 \pm 1,14 \mathrm{~b}$ & $68,00 \pm 1,28 \mathrm{a}$ & 0,0021 \\
Neutrófilos $(\%)$ & $20,00 \pm 1,25 \mathrm{a}$ & $21,50 \pm 0,90 \mathrm{a}$ & $28,58 \pm 1,50 \mathrm{~b}$ & $22,08 \pm 1,73 \mathrm{a}$ & 0,0028 \\
Eosinófilos $(\%)$ & $6,25 \pm 0,50 \mathrm{a}$ & $6,00 \pm 0,27 \mathrm{a}$ & $8,33 \pm 0,62 \mathrm{~b}$ & $6,16 \pm 1,01 \mathrm{a}$ & 0,0074 \\
Monócitos $(\%)$ & $2,33 \pm 0,52$ & $2,75 \pm 0,25$ & $1,83 \pm 0,76$ & $2,16 \pm 0,38$ & 0,2557 \\
Basófilos $(\%)$ & $0,58 \pm 0,38$ & $0,33 \pm 1,44$ & $0,41 \pm 0,52$ & $0,35 \pm 0,25$ & 0,7013 \\
\hline
\end{tabular}

Médias seguidas por letras distintas na linha diferem entre si pelo teste de Wilcoxon-Mann-Whitney $(\mathrm{P}<0,05)$. CTH= contagem total de hemácias $-\mathrm{CTL}=$ contagem total de leucócitos.

Tabela 4. Médias do hematócrito e contagem total e diferencial das células sanguíneas de filhotes de ratas com 10 dias de nascidos

\begin{tabular}{cccccc}
\hline Grupos & I & II & III & IV & P \\
\hline CTH $\left(10^{6} / \mu \mathrm{L}\right)$ & $210,88 \pm 18,76$ & $211,77 \pm 25,61$ & $193,10 \pm 20,22$ & $218,55 \pm 18,59$ & 0,8620 \\
CTL $\left(10^{3} / \mu \mathrm{L}\right)$ & $56,44 \pm 5,21$ & $52,33 \pm 3,17$ & $55,88 \pm 3,68$ & $54,44 \pm 5,01$ & 0,4554 \\
Hematócrito $(\%)$ & $35,88 \pm 2,58$ & $34,86 \pm 3,56$ & $34,33 \pm 0,88$ & $35,11 \pm 2,83$ & 0,9091 \\
Linfócitos $(\%)$ & $73,13 \pm 3,10$ & $74,96 \pm 3,35$ & $73,90 \pm 2,57$ & $77,96 \pm 2,32$ & 0,3806 \\
Neutrófilos $(\%)$ & $15,38 \pm 2,05$ & $16,93 \pm 3,95$ & $18,10 \pm 3,14$ & $16,56 \pm 2,23$ & 0,6762 \\
Eosinófilos (\%) & $8,90 \pm 1,58$ & $7,66 \pm 2,08$ & $7,36 \pm 0,57$ & $8,33 \pm 1,52$ & 0,6359 \\
Monócitos (\%) & $1,43 \pm 0,80$ & $1,23 \pm 1,20$ & $0,46 \pm 0,40$ & $1,00 \pm 0,70$ & 0,5475 \\
Basófilos $(\%)$ & $0,53 \pm 0,20$ & $0,37 \pm 0,22$ & $0,40 \pm 0,36$ & $0,26 \pm 0,15$ & 0,7358 \\
\hline
\end{tabular}

Não houve diferença entre os grupos $(\mathrm{P}>0,05)$ pelo teste de Wilcoxon-Mann-Whitney. $\mathrm{CTH}=$ contagem total de hemácias - CTL $=$ contagem total de leucócitos.

A análise histológica mostrou que o baço dos filhotes com um dia de nascidos de todos os grupos experimentais apresentou parênquima constituído apenas pela polpa vermelha contendo cordões esplênicos ou de Billroth e, entre esses, capilares sinusoides, seios esplênicos e células hematopoiéticas (Fig. 1A e 1B). A partir do quinto dia de nascimento, observou-se a formação da polpa branca caracterizada por nódulos linfáticos ou corpúsculos esplênicos esféricos com arteríola central (Fig. 1C e 1D). Entretanto, no grupo em que as matrizes foram submetidas à pinealectomia e tratadas com placebo, os corpúsculos esplênicos apresentaram forma irregular em comparação aos outros grupos experimentais (Fig. 1E e 1F). Morfometricamente, o baço dos filhotes desse grupo apresentou redução significativa do número de células hematopoiéticas com um e cinco dias de nascidos (Tab. 5 e 6), e na área, diâmetro máximo e mínimo dos corpúsculos esplênicos apenas com cinco dias de nascidos (Tab. 6 e 7). 

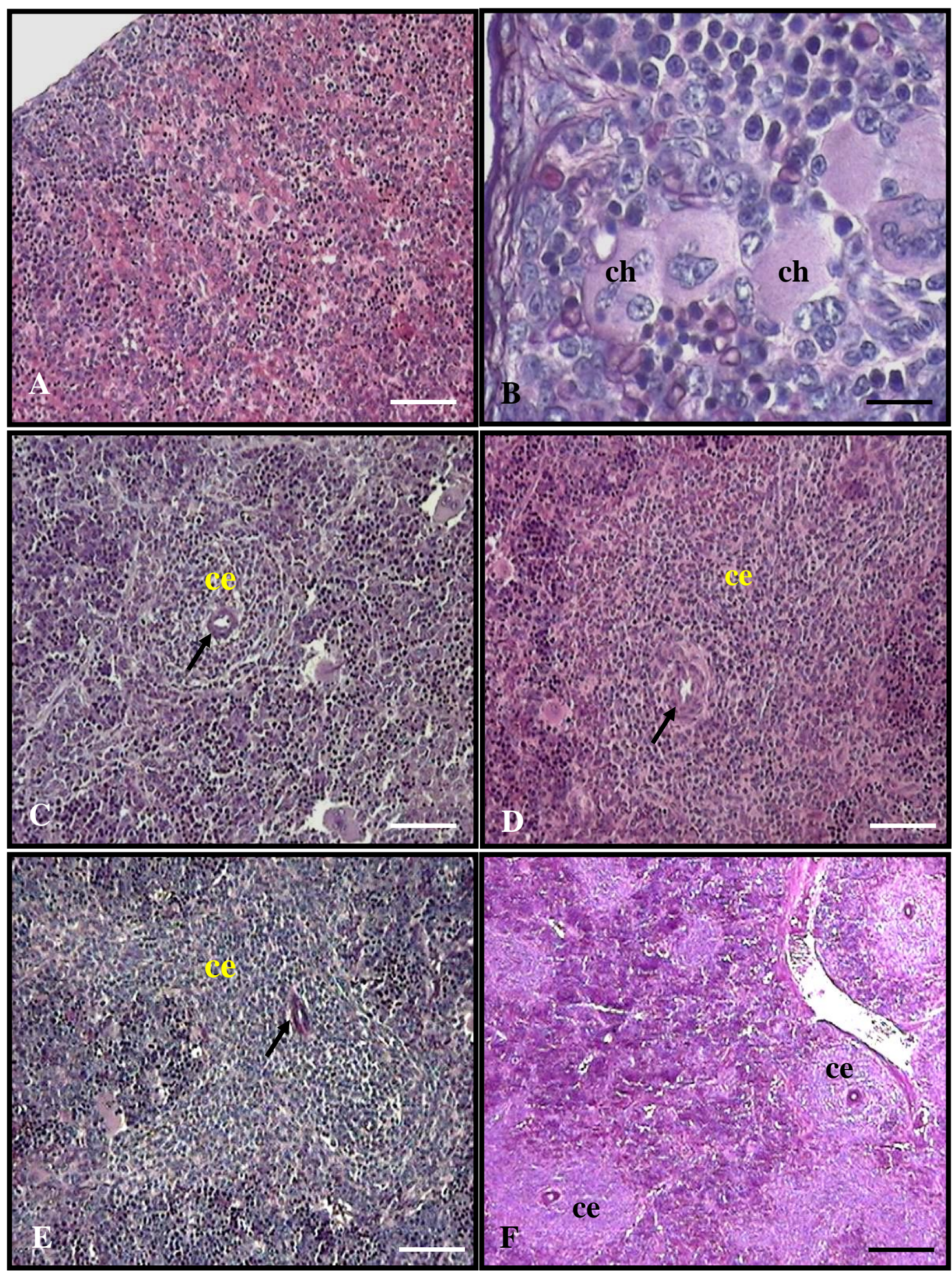

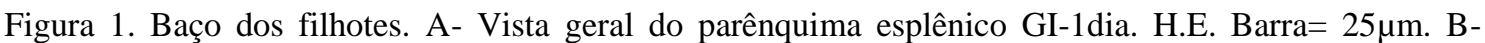
Células hematopoiéticas (ch) GII-5dias. H.E. Barra= 25 $\mu$ m. C, D, E e F -Corpúsculo esplênico (ce) com arteríola central (seta) aos 10 dias de nascimento nos grupos experimentais I, II, III e IV, respectivamente. H.E. Barra $=100 \mu \mathrm{m}$. 
Tabela 5. Médias das características morfométricas do baço e do timo dos filhotes de ratas com um dia de nascidos

\begin{tabular}{|c|c|c|c|c|c|c|}
\hline \multicolumn{7}{|l|}{ Baço } \\
\hline \multicolumn{2}{|l|}{ Grupo } & I & II & III & IV & $\mathrm{P}$ \\
\hline \multicolumn{2}{|l|}{ Área $\left(\mu m^{2}\right)$} & $*$ & $*$ & $*$ & $*$ & - \\
\hline \multicolumn{2}{|c|}{ Diâmetro máximo $(\mu \mathrm{m})$} & $*$ & $*$ & $*$ & $*$ & - \\
\hline \multicolumn{2}{|c|}{ Diâmetro mínimo (um) } & $*$ & $*$ & * & * & - \\
\hline \multicolumn{2}{|c|}{ Células hematopoiéticas } & $15,83 \pm 2,12 \mathrm{a}$ & $14,33 \pm 3,95 \mathrm{a}$ & $9,66 \pm 1,52 b$ & $17,23 \pm 1,50 \mathrm{a}$ & 0,0262 \\
\hline \multicolumn{7}{|l|}{ Timo } \\
\hline Grupo & I & & II & III & IV & $\mathrm{P}$ \\
\hline Área tímica $\left(\mu \mathrm{m}^{2}\right)$ & 134 & $57 \pm 234,76 a$ & $1567,89 \pm 326,09 a$ & $906,41 \pm 240,28 b$ & $1467,43 \pm 249,81 \mathrm{a}$ & 0,0398 \\
\hline $\begin{array}{l}\text { Área cortical } \\
\left(\mu \mathrm{m}^{2}\right)\end{array}$ & 576 & $8 \pm 76,44 a$ & $498,05 \pm 65,90 \mathrm{a}$ & $278,72 \pm 56,91 b$ & $550,06 \pm 59,44 a$ & 0,0196 \\
\hline $\begin{array}{l}\text { Área medular } \\
\left(\mu m^{2}\right)\end{array}$ & 255 & $0 \pm 66,41 \mathrm{a}$ & $203,87 \pm 50,77 a$ & $114,65 \pm 31,09 b$ & $243,55 \pm 49,12 \mathrm{a}$ & 0,0056 \\
\hline
\end{tabular}

Médias seguidas por letras distintas na linha diferem entre si pelo teste de Wilcoxon-Mann-Whitney $(\mathrm{P}<0,05) .{ }^{*}$ Não apresentaram corpúsculos esplênicos.

Tabela 6. Médias das características morfométricas do baço e do timo dos filhotes de ratas com cinco dias de nascidos

\begin{tabular}{|c|c|c|c|c|c|c|c|c|}
\hline \multicolumn{9}{|l|}{ Baço } \\
\hline \multicolumn{3}{|c|}{ Grupo } & \multicolumn{2}{|l|}{$\mathrm{I}$} & II & III & IV & $\mathrm{P}$ \\
\hline \multicolumn{3}{|c|}{ Área $\left(\mu \mathrm{m}^{2}\right)$} & \multicolumn{2}{|c|}{$178,22 \pm 22,89 a$} & $169,61 \pm 19,55 a$ & $239,83 \pm 15,03 b$ & $173,66 \pm 16,39$ & 0,0249 \\
\hline \multicolumn{3}{|c|}{ Diâmetro máximo $(\mu \mathrm{m})$} & \multicolumn{2}{|l|}{$67,88 \pm 9,02 \mathrm{a}$} & $63,00 \pm 11,98 a$ & $98,76 \pm 10,08 b$ & $59,49 \pm 8,73 a$ & 0,0187 \\
\hline \multicolumn{3}{|c|}{ Diâmetro mínimo $(\mu \mathrm{m})$} & \multicolumn{2}{|l|}{$63,15 \pm 10,76 a$} & $60,66 \pm 7,21 a$ & $35,39 \pm 9,87 b$ & $57,63 \pm 7,05 a$ & 0,0492 \\
\hline \multicolumn{3}{|c|}{ Células hematopoiéticas } & $10,50 \pm 1,50 \mathrm{a}$ & & $11,83 \pm 0,95 \mathrm{a}$ & $7,66 \pm 1,43 b$ & $13,05 \pm 2,50 \mathrm{a}$ & 0,0166 \\
\hline \multicolumn{9}{|l|}{ Timo } \\
\hline \multicolumn{2}{|l|}{ Grupo } & I & & II & & III & IV & $\mathrm{P}$ \\
\hline \multicolumn{2}{|c|}{ Área tímica $\left(\mu \mathrm{m}^{2}\right)$} & 376 & $, 52 \pm 167,00 \mathrm{a}$ & & $46,88 \pm 149,37 \mathrm{a}$ & $2976,31 \pm 112,63 b$ & $3497,98 \pm 166,05 a$ & 0,0022 \\
\hline $\begin{array}{l}\text { Área } \\
\left(\mu \mathrm{m}^{2}\right)\end{array}$ & cortical & 197 & $, 55 \pm 114,04 \mathrm{a}$ & & $54,41 \pm 125,73 a$ & $866,02 \pm 155,99 b$ & $1798,40 \pm 119,88 \mathrm{a}$ & 0,0153 \\
\hline $\begin{array}{l}\text { Área } \\
\left(\mu \mathrm{m}^{2}\right)\end{array}$ & medular & 564 & $99 \pm 53,61 \mathrm{a}$ & & $9,76 \pm 49,83 a$ & $332,89 \pm 50,11 \mathrm{~b}$ & $588,27 \pm 47,43 a$ & 0,0066 \\
\hline
\end{tabular}

Tabela 7. Médias das características morfométricas do baço e do timo dos filhotes de ratas com 10 dias de nascidos

\begin{tabular}{|c|c|c|c|c|c|c|}
\hline \multicolumn{7}{|l|}{ Baço } \\
\hline \multicolumn{2}{|l|}{ Grupo } & I & II & III & IV & $\mathrm{P}$ \\
\hline \multicolumn{2}{|l|}{ Área $\left(\mu \mathrm{m}^{2}\right)$} & $322,78 \pm 29,06 a$ & $309,77 \pm 25,91 a$ & $299,35 \pm 22,07 a$ & $313,17 \pm 31,54 a$ & 0,4732 \\
\hline \multicolumn{2}{|c|}{ Diâmetro máximo $(\mu \mathrm{m})$} & $87,55 \pm 10,02 \mathrm{a}$ & $76,39 \pm 9,77 \mathrm{a}$ & $88,45 \pm 11,02 \mathrm{a}$ & $89,66 \pm 8,98 \mathrm{a}$ & 0,2807 \\
\hline \multicolumn{2}{|c|}{ Diâmetro mínimo $(\mu \mathrm{m})$} & $85,15 \pm 7,60 \mathrm{a}$ & $73,21 \pm 6,98 \mathrm{a}$ & $84,07 \pm 8,51 \mathrm{a}$ & $88,38 \pm 9,81 \mathrm{a}$ & 0,1906 \\
\hline \multicolumn{2}{|c|}{ Células hematopoiéticas } & $8,75 \pm 2,71 \mathrm{a}$ & $10,65 \pm 2,07 \mathrm{a}$ & $6,92 \pm 2,86 \mathrm{~b}$ & $9,22 \pm 2,74 \mathrm{a}$ & 0,0011 \\
\hline \multicolumn{7}{|l|}{ Timo } \\
\hline Grupo & I & & II & III & $\mathrm{V}$ & $\mathrm{P}$ \\
\hline Área tímica $\left(\mu \mathrm{m}^{2}\right)$ & & $6,66 \pm 144,00 \mathrm{a}$ & $5461,56 \pm 137,49 a$ & $5227,98 \pm 163,12 a$ & $5387,45 \pm 165,70 a$ & 0,1437 \\
\hline Área cortical $\left(\mu \mathrm{m}^{2}\right)$ & & $4,31 \pm 124,66 a$ & $2375,51 \pm 155,22 \mathrm{a}$ & $2298,76 \pm 135,08 \mathrm{a}$ & $2543,80 \pm 139,21 \mathrm{a}$ & 0,3428 \\
\hline Área medular $\left(\mu \mathrm{m}^{2}\right)$ & & $65 \pm 65,13 a$ & $724,99 \pm 51,01 \mathrm{a}$ & $698,58 \pm 57,04 a$ & $713,67 \pm 62,80 \mathrm{a}$ & 0,2966 \\
\hline
\end{tabular}

Médias seguidas por letras distintas na linha diferem entre si pelo teste de Wilcoxon-Mann-Whitney $(\mathrm{P}<0,05)$.

O timo dos filhotes com um dia, nascidos de ratas pinealectomizadas e tratadas com placebo, apresentou-se menos desenvolvido, quando comparado ao dos outros grupos experimentais, com poucas lobulações contendo zonas cortical e medular e espesso revestimento de tecido mesenquimal (Fig. 2A, 2B e 2C). Essas características mantiveram-se até o quinto dia de 
nascimento (Fig. 2D, 2E e 2F). Aos 10 dias após o nascimento, os timos apresentaram características histológicas similares, porém foi observada a formação do corpúsculo de Hassal nos animais do grupo III (Fig. 3A e 3B). A análise morfométrica mostrou redução significativa nas áreas tímica, cortical e medular apenas nos filhotes do grupo III com um e cinco dias de nascidos (Tab. 5, 6 e 7).
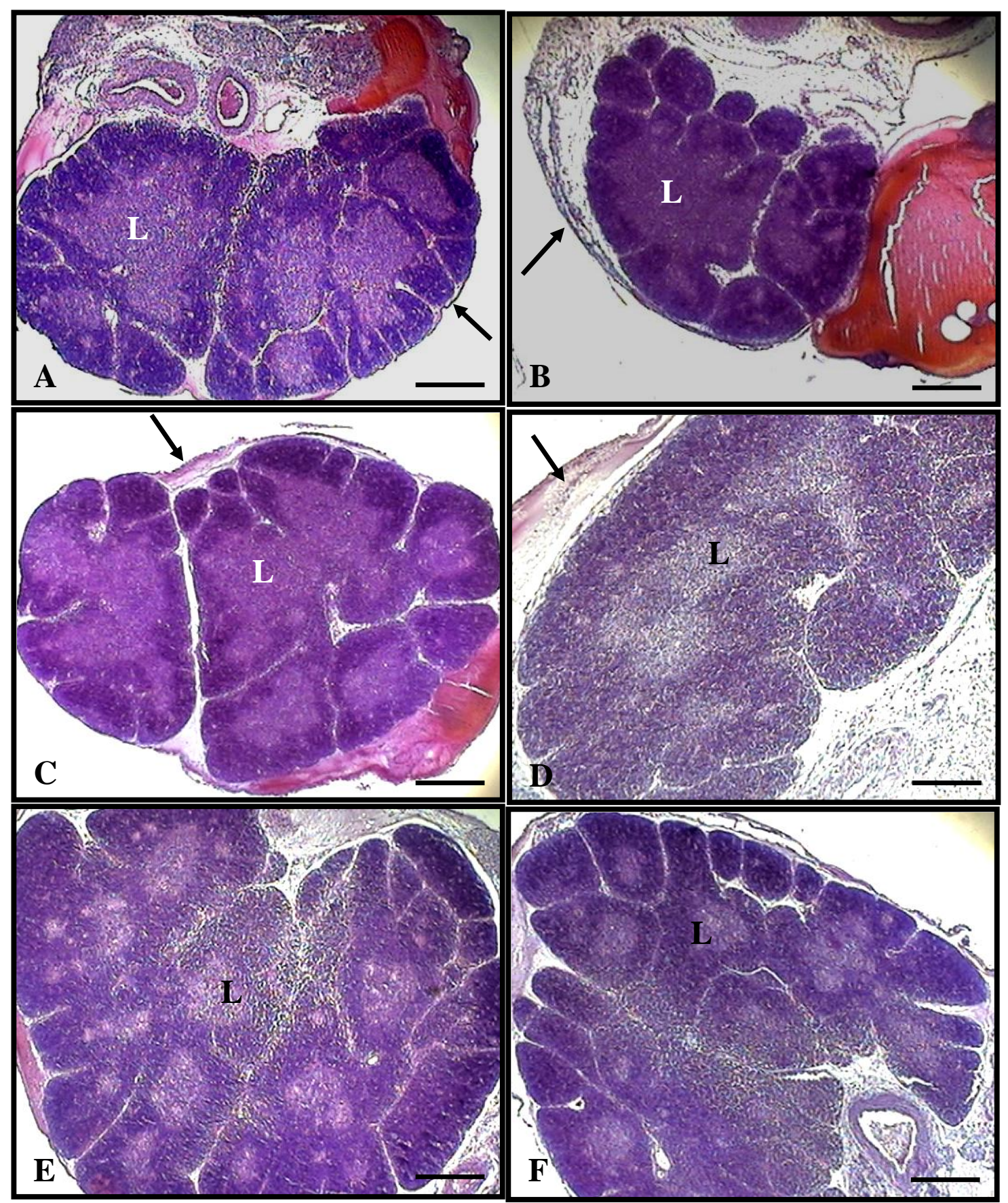

Figura 2. Timo dos filhotes. A, B e C - Lóbulos tímicos (L) em diferentes estágios de desenvolvimento com 1 dia de nascidos nos grupos experimentais I, III e IV, respectivamente. H.E. Barra= 100 $\mu$ m. D lóbulo tímico com 5 dias de nascidos do grupo III mostrando-se ainda pouco desenvolvido em relação aos observados nos grupos II (E) e IV (F). H.E. Barra= 100 $\mu \mathrm{m}$. 
A análise histoquímica não revelou alterações no estroma do baço e do timo referente aos parâmetros: fibras colágenas, elásticas e reticulares (Tab. 8). Em todos os grupos
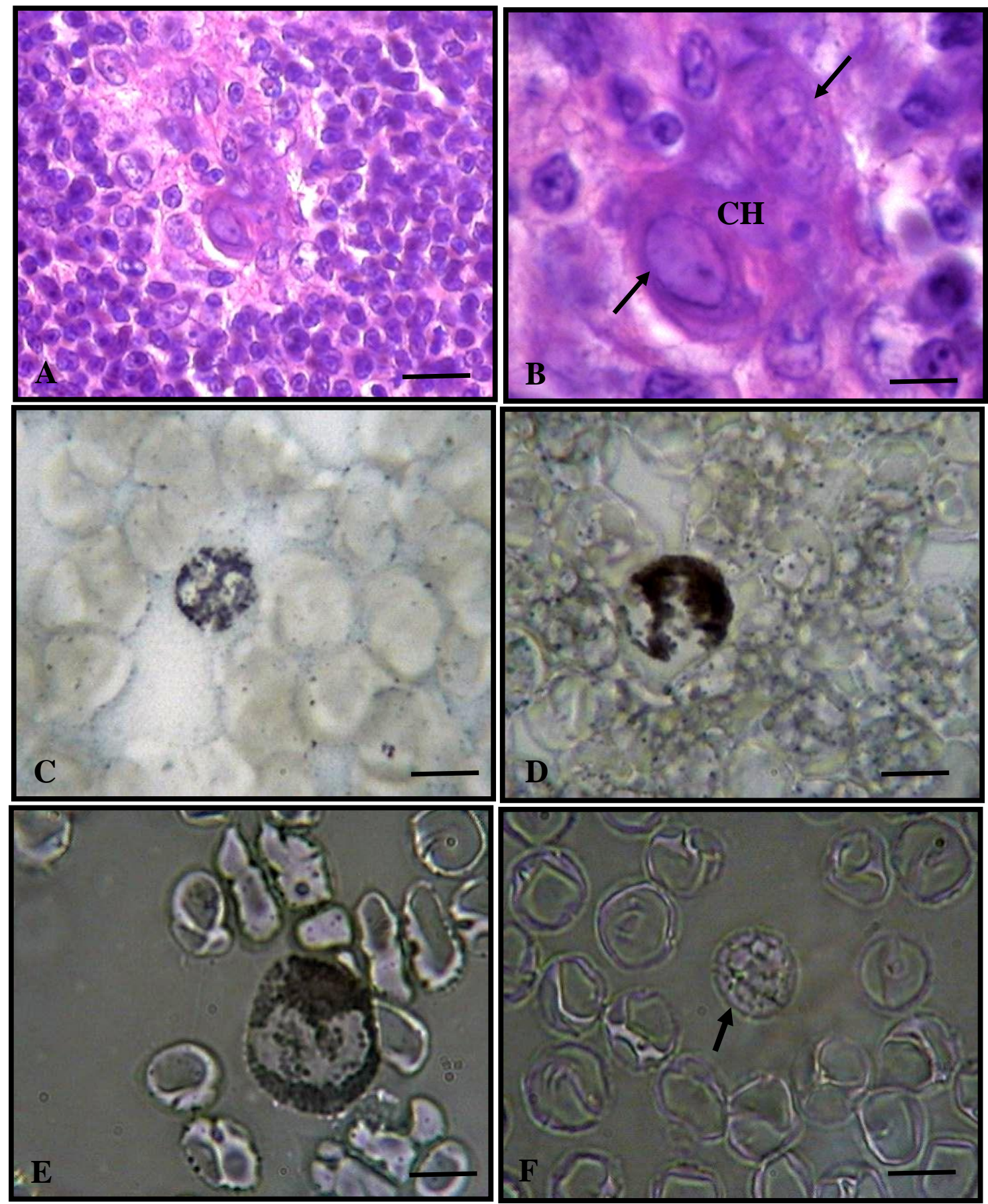

Figura 3. Timo dos filhotes com 10 dias de vida do G III. A - Vista geral do corpúsculo de Hassal. H. E. Barra $=25 \mu \mathrm{m}$. B - Células reticuloepiteliais (setas) compondo o corpúsculo de Hassal. H. E. Barra = $100 \mu \mathrm{m}$. Sangue materno corado pelo Sudam Black 1,4\%. C - Neutrófilo, D - Eosinófilo, D - Monócito, e em F - Linfócito (seta). Barras $=100 \mu \mathrm{m}$. 
Tabela 8. Histoquímica do baço e do timo dos filhotes de ratas dos grupos experimentais

\begin{tabular}{|c|c|c|c|c|}
\hline & GI & GII & GIII & GIV \\
\hline & Baço Timo & Baço Timo & Baço Timo & Baço Timo \\
\hline Fibras colágenas & $\pm \quad \pm$ & $\pm \quad \pm$ & $\pm \quad \pm$ & $\pm \quad \pm$ \\
\hline Fibras elásticas & $+\quad+$ & $+\quad+$ & $+\quad+$ & + \\
\hline Fibras reticulares & $++\quad++$ & $++\quad++$ & $++\quad++$ & $++\quad++$ \\
\hline
\end{tabular}

Reação intensa (++), moderada ( \pm ) e fraca (+)

\section{DISCUSSÃO}

A redução do percentual do hematócrito nas fêmeas com 21 dias de gestação do grupo pinealectomizado tratado com placebo pode estar associada à ausência da melatonina, pois, de acordo com Ozmerdivenli et al. (2011), esse hormônio previne alterações nos parâmetros sanguíneos, inclusive a redução do percentual de hematócrito, produzidos pela exposição à irradiação. A diminuição do número total de leucócitos, caracterizada pelo baixo percentual de linfócitos, embora tenham sido verificadas neutrofilia e eosinofilia, sugere que a melatonina pode ter ação modulatória diferente para esses tipos de leucócitos. Entre as funções que a melatonina desempenha no sistema imune, a habilidade de inibir a apoptose é considerada o seu maior mecanismo (Paternoster et al., 2009). Estudos mostraram que linfócitos $\mathrm{T}$ e $\mathrm{B}$ são induzidos ao processo autóptico em decorrência do estresse oxidativo, o qual aumenta com a aproximação do parto e é agravado pela pinealectomia, porém pode ser revertido pela administração de melatonina, o que indica a importância desse hormônio para a integridade dos linfócitos (Lialiaris et al., 2008; Tamura et al., 2008; Paternoster et al., 2009). De forma diferente, a melatonina tem sido relacionada com a redução de neutrófilos em recém-nascidos (Gitto et al., 2001) e com a apoptose dessas células em algumas patologias (Chen et al., 2003; 2005). No entanto, a melatonina tem mostrado induzir a eosinofilia, por potencializar a ação da interleucina -2 (IL2) (Williamson et al., 1998), e atuar no controle da liberação da peroxidase pelos eosinófilos durante a fagocitose (Lu et al., 2008). O presente estudo mostrou eosinofilia na ausência da melatonina, o que pode estar relacionado aos elevados níveis de estrógeno observados em ratas após pinealectomia (Oztekin et al., 2006), uma vez que os eosinófilos são estimulados por esse esteroide (Ramos et al., 2000). A reação positiva para mieloperoxidase apenas para os neutrófilos, eosinófilos e monócitos está dentro da normalidade, pois quando não há reação nessas células caracteriza-se estados pré-leucêmicos, enquanto reações positivas nos linfócitos determinam a reticuloendoteliose leucêmica (Silva et al., 2006).

A presença de corpúsculos esplênicos com forma irregular e com redução dos diâmetros máximo e mínimo indica que a ausência da melatonina materna pode ter levado à diminuição do número de células desse órgão e consequentemente à desorganização do seu parênquima, pois animais que foram submetidos à iluminação constante (pinealectomia artificial) ou que receberam injeções de bloqueadores para inibir a síntese de melatonina apresentaram diminuição do peso e da celularidade do baço, o que pode ser revertido pela reposição desse hormônio (Rai et al., 2009). A presença das células hematopoiéticas no baço revela sua capacidade mielopoiética, ou seja, a propriedade de gerar os glóbulos vermelhos, a qual tende a diminuir e tornar-se inativa no decurso da idade do animal (Slayton et al., 2002; Pacheco et al., 2003), porém a pinealectomia parece acelerar esse processo.

O menor desenvolvimento do timo dos filhotes com um e cinco dias de idade, provenientes das fêmeas do grupo pinealectomizado e tratado com placebo, foi confirmado pelas análises morfométricas. Estudos mostraram atrofia do timo com diminuição do peso em ratos machos adultos, além da ausência de distinção entre as zonas cortical e medular, bem como a perda de elementos linfoides após a pinealectomia (Öner et al., 2004).

Os resultados apontam para a importância da melatonina no desenvolvimento e na manutenção do timo em ratos neonatos, pois mesmo não havendo alterações morfométricas no timo dos filhotes com 10 dias de idade, a presença do corpúsculo de Hassal indica processo de maturação tímica precoce pelo fato de essa 
estrutura estar envolvida na atividade regulatória dos linfócitos T (Berthelot et al., 2010). Por outro lado, o presente estudo mostrou que a ausência da melatonina materna não teve nenhuma influência sobre o baço e o timo da prole aos 10 dias de idade, o que pode estar relacionado ao fato de que em ratos a síntese de melatonina tem início entre o quinto e sétimo dias de vida, quando é estabelecida a inervação da pineal (Pfeffer e Stehle, 1998).

\section{CONCLUSÕES}

A melatonina tem importante função na modulação do hemograma em ratas na última semana de gestação, e a transferência de melatonina materna para a prole é de grande relevância para garantir o desenvolvimento normal do baço e do timo nos primeiros dias de vida.

\section{REFERÊNCIAS}

ALVES, C.M.; VASCONCELOS, A.C.; MARTINS, A.S. et al. Morphometric analysis of the thymus of puppies infected with the Snyder Hill Strain of canine distemper virus. Arq. Bras. Med. Vet. Zootec., v.58, p.472-479, 2006.

BALEY, J.E.; SCHACTER, B.Z. Mechanisms of diminished natural killer cell activity in pregnant women and neonates. J. Immunol., v.134, p.30423048, 1985

BERTHELOT, J.M.; GOFF, B.; MAUGARS, Y. Thymic Hassall's corpuscle, Regulatory T-Cells, and Rheumatoid Arthritis. Semin. Arthritis Rheu., v.39, p.347-355, 2010.

BISHNUPURI, K.S.; HALDAR, C. Maternal transfer of melatonin alters tho growth and sexual maturation of young Indian palm squirrel Funambulus pennanti. Biol. Signals Recept., v.10, p.317-325, 2001.

CHEN, J-C.; NG, C-H.; CHIU, T-F.; CHEN, H-M. Altered neutrophil apoptosis activity is reversed by melatonin in liver ischemia-reperfusionJ. J. Pineal Res., v.34, p.260-264, 2003

CHEN, H-M.; HSU, J-T.; CHEN, J-C. et al. Delayed Neutrophil apoptosis attenuated by melatonin in human acute pancreatitis. Pancreas, v.31, p.360-364, 2005.

CLAUSTRAT, T.B.; BRUN, J.; CHAZOT, G. The basic physiology and pathophysiology of melatonin. Sleep Med. Rev., v.9, p.11-24, 2005.
EL-SOKKARY, G.H.; REITER, R.J.; ABDELGHAFFAR, S.K.H. Melatonin supplementation restores cellular proliferation and DNA synthesis in the splenic and thimic lymphocytes of old rats. Neuroendocrinol. Lett., v.24, p.215-223, 2003.

ESQUIFINO, A.I.; CHACON, F.; CANO, P. et al. Twenty-four-hour rhythms of mitogenic responses, lymphocyte subset populations and amino acid content in submaxillary lymph nodes of growing male rats subjected to calorie restriction. J. Neuroimmunol., v.156, p.66-73, 2004

FAAS, M.M.; BOUMAN, A.; NIEUWENHOVEN, A.L.V.V. et al. Species differences in the effect of pregnancy on lymphocyte cytokine production between human and rat. J. Leukocyte Biol., v.78, p.946-953, 2005.

GABRILOVAC, J.; ZADJELOVIC, J.; OSMAK, M. et al. NK cell activity and estrogen hormone levels during normal human pregnancy. Gynecol. Obstet. Inves., v.25, p.165-172, 1988.

GITTO, E.; KARBOWNIK, M.; REITER, J.R. et al. Effects of melatonin treatment in septic newborns. Pediatr. Res., v.50, p.756-760, 2001.

GOLALIPOUR, M.J.; KORD, H.; GHAFARI, S. et al. Morphometric alterations to the rat spleen following formaldehyde exposure. Folia Morphol., v.67, p.6671, 2008.

GOLDMAN, B.D. Pattern of melatonin secretion mediates transfer of photoperiod information from mother to fetus in mammals. Science's STKE., v.192, p.29-31, 2003

KUSZAK, J.; RODIN, M. A new technique of pinealectomy for adult rats. Experientia, v.33, p.283284, 1977.

LIALIARIS, T.; LYRATZOPOULOS, E.; PAPACHRISTOU, F. et al. Supplementation of melatonin protects human lymphocytes in vitro from the genotoxic activity of melphalan. Mutagenesis, v.23, p.347-354, 2008.

LORENZI, T. Manual de hematologia: propedêutica e clínica. 4.ed. São Paulo: Guanabara Koogan, 2006. $722 \mathrm{p}$.

LU, T.; GALIJASEVIC, S.; ABDULHAMID, I.; ABU-SUOD, H.M. Analysis of the mechanism by which melatonin inhibits human eosinophil peroxidase. Br. J. Pharmacol., v.154, p.1308-1317, 2008.

MARKERT, U.R.; FITZGERALD, J.S.; SEYFARTH, L. et al. Lessons from reproductive immunology for other fields of immunology and clinical approaches. Chem. Immunol. Allergy, v.89, p.169-179, 2005. 
NAKAMURA, Y.; TAMURA, H.; KASHIDA, S. et al. Changes of serum melatonin level and its relationship to feto-placental unit during pregnancy. $J$. Pineal Res., v.30, p.29-33, 2001.

ÖNER, H.; KUS, I.; ÖNER, J. et al. Possible effects of melatonin on thymus gland after pinealectomy in rats. Neuroendocrinol. Lett., v.25, p.115-118, 2004.

OZMERDIVENLI, R.; KARACABEY, K.; GUNDOGDU, C. et al. Protective role of melatonin on blood parameters following irradiation in rat. Afr. $J$. Biotechnol., v.10, p. 18564-18568, 2011.

OZTEKIN, E.; MOGULKOC, R.; BALTACI, A.K.; TIFTIK, A.M. The influence of estradiol and progesterone and melatonin supplementation on TNFalpha levels in ovariectomized and pinealectomized rats. Acta Biol. Hung., v.57, p.275-281, 2006.

PACHECO, M.R.; NAKAGHI, L.S.O.; ARTONI, S.M.B. et al. Morfologia da megacariocitopoiese esplenica em coelhos da raca Nova Zelandia Branco, no final da gestação e pos-natal. Acta Sci., v.25, p.109114, 2003.

PATERNOSTER, L.; RADOGNA, F.; ACCORSI, A. et al. Melatonin as a Modulator of Apoptosis in BLymphoma Cells. Ann. NY Acad. Sci., v.1171, p.345349, 2009.

PERTSOV, S. Effect of melatonin on the thymus, adrenal glands, and spleen in rats during acute stress. B. Exp. Biol. Med., v.141, p.292-295, 2006.

PFEFFER, M.; STEHLE J.H. Ontogeny of a diurnal rhythm in arylalkylamine- $\mathrm{N}$-acetyltransferase mRNA in rat pineal gland. Neurosci. Lett., v.248, p.163-166, 1998.

RAI, S.; HALDAR, C.; SINGH, R. Modulation of immunity in young-adult and aged squirrel, Funambulus pennati by melatonin and pchlorophenylalanine. Immun. Ageing., v.6, p.1-8, 2009.

RAMOS, J.G.; VARAYOUD, J.; KASS, L. et al. Estrogen and progesterone modulation of eosinophilic infiltration of the rat uterine cervix. Steroids, v.65, p.409-414, 2000.

SARGENT, I.L.; BORZYCHOWSKI, A.M.; REDMAN, C.W. NK cells and human pregnancy - an inflammatory view. Trends Immunol., v.27, p.399404, 2006.
SHAKHAR, K.; VALDIMARSDOTTIR, H.B.; HEIGHTENED, D.H.B. Risk of breast cancer following pregnancy: could lasting systemic immune alterations contribute? Cancer Epidem. Biomar., v.16, p.1082-1086, 2007.

SILVA, G.C.; PILGER, D.A.; CASTRO, S.M.; WAGNER, S.C. Diagnóstico laboratorial das leucemias mieloides agudas. J. Bras. Patol. Med. Lab., v.42, p.77-84, 2006.

SLAYTON, W.B.; GEORGELAS, A.L.; PIERCE, J. et al. The spleen is a major site of megakaryopoiesis following transplantation of murine hematopoietic stem cells. Blood, v.100, p.3975-3982, 2002.

SOMERSET, D.A.; ZHENG, Y.; KILBY, M.D. et al. Normal human pregnancy is associated with an elevation in the immune suppressive CD25+ CD4+ regulatory T-cell subset. Immunology, v.112, p.38-43, 2004.

SUBRAMANIAN, P.; MIRUNALINI, S.; PANDIPERUMAL, S.R. et al. Melatonin treatment improves the antioxidant status and decreases lipid content in brain and liver of rats. Eur. J. Pharmacol., v.571, p.116-119, 2007.

TAMURA, H.; NAKAMURA, Y.; TERRON, M.P. et al. Melatonin and pregnancy in the human. Reprod. Toxicol., v.25, p.291-303, 2008.

VEENSTRA VAN NIEUWENHOVEN, A.L.; HEINEMAN, M.J.; FAAS, M.M. The immunology of successful pregnancy. Hum. Reprod. Update, v.9, p.347-357, 2003.

WEIBEL, E.R.; KISTLER, G.S.; SCHERLE, W.F. Practical stereological methods for morphometric cytology. J. Cell Biology, v.30, p.23-38, 1966.

WILLIAMSON, B.L.; TOMLINSON, A.J.; MISHRA, P.K. et al. Related compounds from L-tryptophan associated with eosinophilia-myalgia syndrome. Chem. Res. Toxicol., v.11, p.234-240, 1998.

WRONKA, M.; MALESZEWSKA, M.; STĘPIŃSKA, U.; MARKOWSKA, M. Diurnal differences in melatonin effect on intracellular $\mathrm{Ca}^{2+}$ concentration in chicken spleen leukocytes in vitro. J. Pineal Res., v.44, p.134-140, 2008. 